\title{
Análise descritiva da expressão de PD-L1 em biopsias de carcinoma de não pequenas células do pulmão: experiência de dois anos em Hospital terciário
}

\section{Descriptive analysis of PD-L1 expression in non-small cell lung carcinoma biopsies: two-year experience in a tertiary Hospital}

\author{
C. Fleming 1,* iD, P. Fidalgo² (iD), H. Miranda² (iD), R. Romão² (iD), A. Araújo² (iD), J. Vizcaíno \\ ${ }^{1}$ Serviço de Anatomia Patológica, Centro Hospitalar e Universitário do Porto, Porto, Portugal \\ ${ }^{2}$ Serviço de Oncologia Médica, Centro Hospitalar e Universitário do Porto, Porto, Portugal \\ *u12643@chporto.min-saude.pt
}

\section{RESUMO}

Introdução: A expressão de PD-L1 como marcador preditivo de resposta ao tratamento do carcinoma pulmonar de não pequenas células (CPNPC) permite identificar os doentes com maior probabilidade de beneficiar de tratamento com inibidores de checkpoints imunológicos. O principal objectivo deste estudo foi determinar as taxas de positividade/negatividade de PD-L1 de biopsias com diagnóstico de CPNPC durante o ano de 2017 e 2018 no CHUPorto e comparar com as taxas previstas.

Materiais e métodos: Procedeu-se ao levantamento de todas as biopsias de pulmão, brônquio ou pleura com diagnóstico de neoplasia primária epitelial e categorizou-se de acordo com a idade e sexo dos doentes, o diagnóstico relatado, o nível de expressão de PD-L1 e o estudo mutacional.

Resultados: Dos 262 casos identificados, $57 \%$ correspondem ao diagnóstico de adenocarcinoma, 26\% a carcinoma epidermóide, $11 \%$ a neoplasia neuroendócrina e $6 \%$ a outros. A expressão de PD-L1 foi avaliada em 215 biopsias das 229 registadas com o diagnóstico de CPNPC. Destes 215 casos, $54 \%$ foram negativos, $26 \%$ revelou positividade ligeira a intermédia e $20 \%$ positividade elevada para PD-L1.

Discussão e conclusão: A avaliação da expressão de PD-L1 em biopsias de carcinomas de origem pulmonar é uma ferramenta essencial para a definição do tratamento destes doentes. Os valores aqui descritos de expressão de PD-L1 na prática clínica do CHUPorto são tendencialmente mais negativos do que os descritos no ensaio clínico original mas semelhantes aos descritos por estudos de vida real. Atendendo ao impacto que a expressão de 
PD-L1 tem na gestão de doentes com CPNPC na prática clínica diária, é relevante conhecermos a nossa realidade. Como perspectivas futuras, consideramos importante a análise de correlação da expressão de PD-L1 com os resultados do tratamento com inibidores dos checkpoints imunológicos.

Palavras-chave: PD-L1, carcinoma não pequenas células, pulmão

(c) 2021 Grupo de Estudos do Cancro do Pulmão. Publicado por Publicações Ciência \& Vida. Este é um artigo Open Access sob uma licença CC BY-NC-ND (http://creativecommons.org/licenses/by-nc-nd/4.0/).

\begin{abstract}
Introduction: The expression of PD-L1 as a predictive marker of response to treatment of non-small cell lung cancer (NSCLC) allows the identification of patients most likely to benefit from treatment with inhibitors of immune checkpoints. The main objective of this study was to determine the PD-L1 positivity/negativity rates of biopsies diagnosed with NSCLC during the year 2017 and 2018 at CHUPorto and compare with the predicted rates.

Materials and methods: All biopsies of lung, bronchus or pleura diagnosed with primary epithelial neoplasia were surveyed and categorized according to the age and sex of patients, the reported diagnosis, the level of expression of PD- L1 and the mutational study.

Results: Of the 262 cases identified, $57 \%$ were diagnosed with adenocarcinoma, $26 \%$ with squamous cell carcinoma, $11 \%$ with neuroendocrine neoplasia and $6 \%$ with others. PD-L1 expression was evaluated in 215 biopsies out of 229 recorded with the diagnosis of NSCLC. Of these 215 cases, $54 \%$ were negative, $26 \%$ showed mild to intermediate positivity and $20 \%$ high positivity for PD-L1.

Discussion and conclusion: The evaluation of PD-L1 expression in lung cancer biopsies is an essential tool for defining the treatment of these patients. The values of PD-L1 expression described here in the clinical practice of CHUPorto tend to be more negative than those described in the original clinical trial, but similar to those described by real-life studies. Given the impact that the expression of PD-L1 has on the management of patients with NSCLC in daily clinical practice, it is important to know our reality. As future perspectives, we consider important the correlation analysis of PD-L1 expression with the results of treatment with inhibitors of immune checkpoints.
\end{abstract}

Keywords: PD-L1, non-small cell carcinoma, lung

(C) 2021 Grupo de Estudos do Cancro do Pulmão. Published by Publicações Ciência \& Vida. This is an open access article under the CC BY-NC-ND license (http://creativecommons.org/licenses/by-nc-nd/4.0/).

\section{INTRODUÇÃO}

A expressão de PD-L1 como marcador preditivo de resposta ao tratamento do carcinoma pulmonar de não pequenas células (CPNPC) permite identificar os subgrupos de doentes que têm maior probabilidade de beneficiar de tera- pêutica com agentes inibidores de checkpoints imunológicos. A sua determinação é feita de forma sistemática na prática clínica diária, uma vez que tem implicações terapêuticas em doentes com doença localmente avançada e metastáti$\mathrm{ca}^{1-7}$. A sua determinação é realizada por anatomopatologistas através de estudo imunohisto-

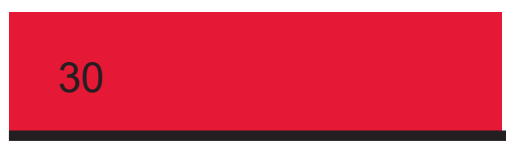


químico. A avaliação do marcador PD-L1 em biopsias de CPNPC no Serviço de Anatomia Patológica (SAP) do Centro Hospitalar e Universitário do Porto (CHUPorto) teve início no final do ano de 2016. Após alguns anos de experiência com este marcador, torna-se necessário avaliar se as taxas de positividade/negatividade deste marcador estão de acordo com as taxas previstas pelos ensaios clínicos que as preconizaram e de acordo com o esperado na prática clínica.

O principal objectivo deste estudo foi determinar as taxas de positividade/negatividade do PD-L1 de biopsias com diagnóstico de CPNPC durante o ano de 2017 e 2018 e comparar com as taxas previstas nos ensaios clínicos.

\section{MATERIAIS E MÉTODOS}

No âmbito deste trabalho, procedeu-se ao levantamento de todas as biopsias de pulmão, brônquio ou pleura com diagnóstico de neoplasia primária epitelial, incluindo adenocarcinoma, carcinoma epidermóide, neoplasia neuroendócrina ou outros carcinomas, que deram entrada no SAP do CHUPorto, nos anos de 2017 e 2018 . Foram identificados 262 casos com estes critérios. As biopsias foram categorizadas de acordo com a idade e sexo dos doentes, o diagnóstico relatado, o nível de expressão de PD-L1 (negativo (0-1\%), positivo ligeiro a intermédio (1-49\%) e positivo elevado $(>50 \%))$ por imunohistoquímica com o anticorpo anti-PD-L1 da DAKO, clone 22C3, e o estudo mutacional, que incluía mutações nos genes EGFR, BRAF e RAS por biologia molecular e o estudo de rearranjos do gene ALK por citogenética. Neste estudo, o nível de expressão do PD-L1 foi determinado, de acordo com as instruções do fabricante do anticorpo ${ }^{8}$, pelo Tumor Proportion Score (TPS), que requer a presença de 100 ou mais células tumorais viáveis, e constitui a razão entre o número de células tumorais positivas e o número total de células tumorais. A positividade é reconhecida como marcação membranar, completa ou incompleta, fraca a intensa. A análise estatística foi realizada com o Microsoft Excel.

\section{RESULTADOS}

A idade média dos doentes da amostra analisada foi de 66 anos, sendo $76 \%$ do sexo masculino e $24 \%$ do sexo feminino. A distribuição do diagnóstico por sexo foi assimétrica, sendo o exemplo mais evidente o do diagnóstico de carcinoma epidermóide que, nos anos de 2017 e 2018, apenas afetou 3 mulheres (Gráfico I).

Gráfico I. Distribuição dos casos por sexo e por diagnóstico em número absoluto

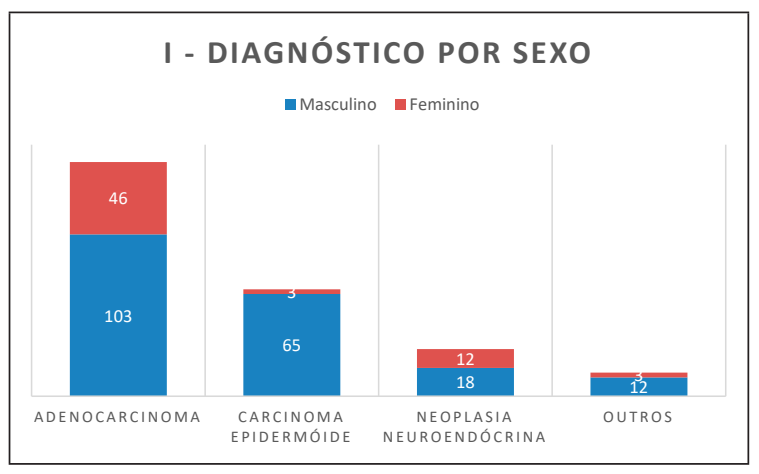

Dos 262 casos identificados, 149 (57\%) correspondem ao diagnóstico de adenocarcinoma, $68(26 \%)$ a carcinoma epidermóide, $30(11 \%)$ a neoplasia neuroendócrina e $15(6 \%)$ a outros carcinomas como por exemplo, carcinoma mucoepidermóide ou carcinoma sem outra especificação (SOE) (Gráfico II). 
Gráfico II. Distribuição dos diagnósticos seleccionados em percentagem

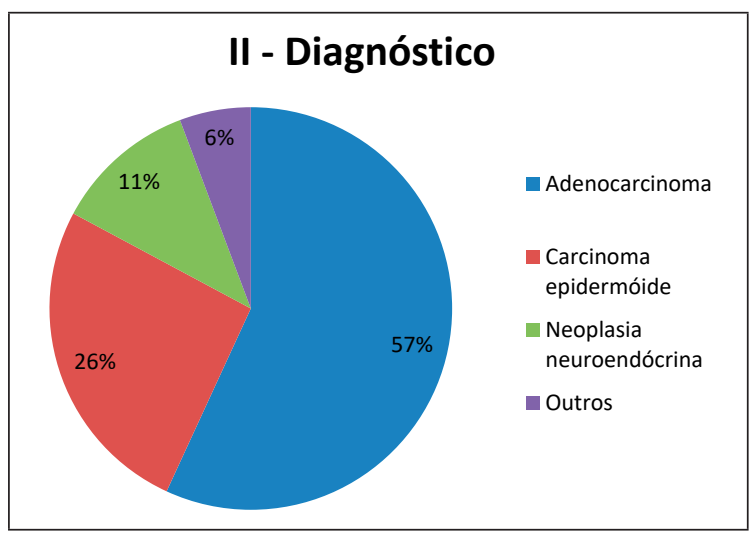

Em relação ao marcador TTF1, constata-se que foi aferido em 22 dos 30 casos de neoplasia neuroendócrina revelando positividade em 17 amostras testadas (77\%). Dos 149 doentes com o diagnóstico de adenocarcinoma, em 137 foi realizado o estudo imunohistoquímico para TTF1, sendo positivo em 112 casos (82\%) e negativo em 25 (18\%).

A expressão de PD-L1 foi avaliada em 215 biopsias das 229 registadas com o diagnóstico de carcinoma de não pequenas células (CPNPC), que inclui os diagnósticos de adenocarcinoma, carcinoma epidermóide e carcinoma SOE (Tabela 1). Destes 215 casos, $54 \%$ foram negativos, $26 \%$
Gráfico III. Distribuição da categoria de PD-L1 por diagnóstico em número absoluto. CNPC - Carcinoma de não pequenas células, SOE - Sem outra especificação

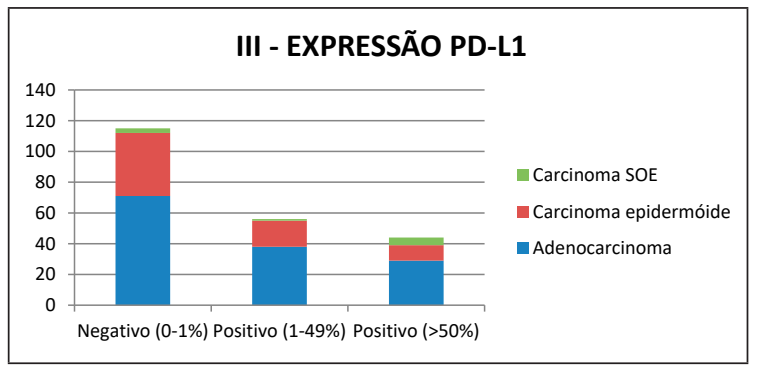

revelou positividade ligeira a intermédia e $20 \%$ positividade elevada para PD-L1 (Gráfico III).

Em 2017 e 2018, o estudo das mutações em casos de adenocarcinoma já se encontrava instituído no SAP do CHUPorto. O painel de estudo mutacional incluía mutações nos genes EGFR, $B R A F$ e RAS por biologia molecular e o estudo de rearranjos do gene $A L K$ por citogenética. Dos 149 casos de adenocarcinoma, o estudo mutacional foi realizado em 133 casos. Foram identificadas mutações em 76 doentes, correspondendo a uma percentagem de $57 \%$ dos casos testados. A mutação RAS foi a mais frequente (47 doentes), seguida da mutação de EGFR (21 doentes), rearranjo de $A L K$ ( 5 doentes) e mutação de BRAF ( 3 doente) (Gráfico IV). Não foram identificadas mutações em 57 casos (43\%).

Tabela 1. Distribuição da avaliação do PD-L1 por diagnóstico em número absoluto e percentagem. CNPC - Carcinoma de não pequenas células, SOE - Sem outra especificação

\begin{tabular}{|c|c|c|c|c|c|c|c|c|c|}
\hline \multicolumn{2}{|c|}{ PD-L1 } & \multicolumn{2}{c|}{ Total CNPC } & \multicolumn{2}{c|}{ Adenocarcinoma } & Carcinoma epidermóide & \multicolumn{2}{c|}{ Carcinoma SOE } \\
\hline Categoria & Intervalo & N. ${ }^{\circ}$ & $\%$ & N. ${ }^{\circ}$ & $\%$ & N..$^{\circ}$ & $\%$ & N. ${ }^{\circ}$ & $\%$ \\
\hline Negativo & $0-1 \%$ & 115 & $54 \%$ & 71 & $51 \%$ & 41 & $60 \%$ & 3 & $33 \%$ \\
\hline Positivo & $1-49 \%$ & 56 & $26 \%$ & 38 & $28 \%$ & 17 & $25 \%$ & 1 & $11 \%$ \\
\hline Positivo & $\geq 50 \%$ & 44 & $20 \%$ & 29 & $21 \%$ & 10 & $15 \%$ & 5 & $56 \%$ \\
\hline
\end{tabular}


Gráfico IV. Estudo mutacional de adenocarcinomas: distribuição em percentagem

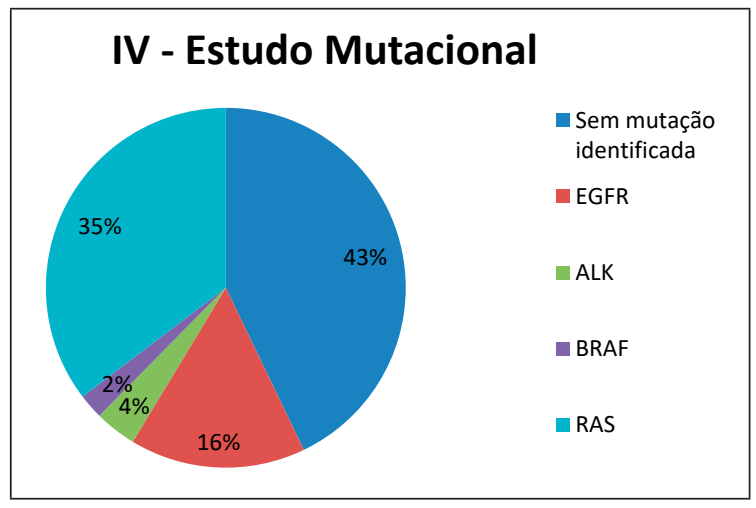

\section{DISCUSSÃO}

A avaliação da expressão de PD-L1 em biopsias de CPNPC é uma ferramenta essencial para a definição do tratamento destes doentes ${ }^{1-7}$. De facto, frequentemente, a única amostra avaliada é o tecido de biopsia, que por definição é exíguo, não existindo acesso à totalidade da neoplasia se o doente não tiver indicação cirúrgica. De lembrar, que a expressão de PD-L1 em carcinomas pulmonares é comprovadamente heterogénea ${ }^{8-9}$, com focos de maior expressão e focos de menor ou até expressão ausente, logo levantando o problema de representatividade da biopsia. A colheita de tecido de biopsia em quantidade suficiente é fulcral, já que estudos anteriores revelaram que a representatividade da biopsia está relacionada com a quantidade de amostra ${ }^{9}$.

Os valores aqui descritos de expressão de PD-L1 na prática clínica do CHUPorto são tendencialmente mais negativos do que os descritos no ensaio clínico KEYTRUDA KEYNOTE-024 em que se baseou a aprovação do anticorpo utilizado no $\mathrm{SAP}^{10}$. A distribuição pelas 3 categorias (negativo, positivo ligeiro a intermédio e positivo elevado) nesse estudo inicial era de $31 \%, 39 \%$ e $30 \%$ respectivamente. Nas biopsias de 2017 e 2018 do CHUPorto, a distribuição pelas 3 categorias (negativo, positivo ligeiro a intermédio e positivo elevado) foi de $54 \%, 26 \%$ e $20 \%$ respectivamente. Outros estudos como o de Munari et al. (2017) demonstraram negatividade em $60 \%$ das biopsias de adenocarcinoma e carcinoma epidermoide. Esta tendência de negatividade poderá estar relacionada com diversos factores, nomeadamente com a representatividade da neoplasia como já referido. Outros factores podem incluir questões técnicas, diferenças intra- e inter-observador e o número de casos existentes. Importante mencionar também que um ensaio clínico por definição controla um conjunto de variáveis que, na prática clínica envolvendo a população em geral, dificilmente é possível. De referir ainda que a intensidade de marcação não é relevante para a categoria de expressão atribuída à biopsia mas pode influenciar inadvertidamente a classificação da mesma, já que uma intensidade forte de marcação é facilmente reconhecida em detrimento de uma intensidade fraca ${ }^{11}$.

A análise da expressão de PD-L1 nesta amostra permite ainda assinalar que, comparando os diagnósticos de adenocarcinoma e carcinoma epidermóide, a distribuição de negatividade e positividade do PD-L1 é semelhante com uma ligeira tendência para os carcinomas epidermóides serem mais frequentemente negativos para PD-L1. Curiosamente, nos carcinomas SOE, os casos positivos fortes foram mais frequentes, mas, dado o número reduzido de casos, não é possível inferir tendências.

Relativamente à expressão do anticorpo TTF1 constata-se que $18 \%$ dos adenocarcinomas são negativos para este marcador, percentagem esta inferior à descrita previamente ${ }^{12}(25 \%)$. Este mar- 
cador é de suma importância no diagnóstico de neoplasias pulmonares contribuindo para aferir se o carcinoma presente no pulmão é primário ou secundário. A maioria dos carcinomas de origem pulmonar é positiva para TTF1 permitindo, juntamente com os dados clínicos e imagiológicos, a definição da origem da neoplasia.

Relativamente às percentagens de frequência das mutações testadas, as descritas neste estudo são consistentes com as referidas em estudos prévios $^{12}$. Actualmente, o estudo molecular de neoplasias é um campo em franco crescimento tornando-se um forte aliado no diagnóstico detaIhado das mesmas. O estudo molecular com identificação de drivers oncogénicos passíveis de terapêutica dirigida a alvos moleculares é fundamental no tratamento da doença avançada, com impacto positivo na sobrevivência nestes doentes quando tratados com terapêutica alvo. Cada vez mais tem existido um investimento para a determinação sistemática destes drivers oncogénicos em doentes com CPNPC não-escamoso, com a utilização preferencial de painéis multigénicos que permitem uma determinação mais alargada destes biomarcadores. Também está descrito o impacto que algumas alterações driver podem ter na resposta à imunoterapia com inibidores dos checkpoints imunológicos ${ }^{13}$, pelo que para a definição da melhor estratégia de tratamento na doença avançada é necessária a integração dos diferentes biomarcadores (PD-L1 e drivers oncogénicos) no algoritmo de decisão clínica.

\section{CONCLUSÃO}

A avaliação da expressão de PD-L1 em carcinomas de origem pulmonar reveste-se de grande interesse na prática clínica. Assim, a aprecia- ção da realidade hospitalar torna-se uma ferramenta importante na melhoria dos cuidados prestados ao doente.

De futuro, seria importante aferir se a taxa de positividade do PD-L1 se correlaciona como prognóstico dos doentes seleccionados para tratamento imunológico, com diagnóstico de CPNPC.

\section{ORCID}

Carolina Fleming (ID) 0000-0003-2106-8744

Paula Fidalgo (iD) 0000-0002-6045-7059

Hugo Miranda (D) 0000-0002-2798-5554

Raquel Romão (iD) 0000-0003-1062-7387

António Araujo (iD) 0000-0002-7267-3584

Ramon Vizcaino (iD) 0000-0003-4548-4179

\section{REFERÊNCIAS}

1. Antonia SJ, Villegas A, Daniel D, et al. Overall Survival with Durvalumab after Chemoradiotherapy in Stage III NSCLC. N Engl J Med. 2018, 379(24):2342-50.

2. Gandhi L, Rodríguez-Abreu D, Gadgeel S, et al. Pembrolizumab plus Chemotherapy in Metastatic Non-Small-Cell Lung Cancer. N Engl J Med. 2018, 378(22):2078-92.

3. Reck M, Rodríguez-Abreu D, Robinson AG, et al. Pembrolizumab versus Chemotherapy for PD-L1-Positive Non-Small-Cell LungCancer. N Engl J Med. 2016, 375(19):1823-33.

4. Herbst RS, Baas P, Kim DW, et al. Pembrolizumab versus docetaxel for previously treated, PD-L1-positive, advanced non-small-cell lungcancer (KEYNOTE-010): a randomized controlled trial. Lancet. 2016, 387(10027):1540-50.

5. Rittmeyer A, Barlesi F, Waterkamp D, et al. Atezolizumab versus docetaxel in patients with previously treated non-small-cell lung cancer (OAK): a phase 3 , open-label, multicentre randomized controlled trial. Lancet. 2017, 389(10066):255-65.

6. Borghaei H, Paz-Ares L, Horn L, et al. Nivolumab versus Docetaxel in Advanced Nonsquamous Non-

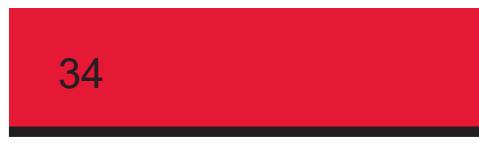


-Small-Cell Lung Cancer. N Engl J Med. 2015, 373(17):1627-39.

7. Planchard D, Popat S, Kerr K, et al. Metastatic non-small cell lung cancer: ESMO Clinical Practice Guidelines for diagnosis, treatment and follow-up. ESMO Guidelines Committee. Ann Oncol. 2018, 29(Suppl 4):192-237

8. PD-L1 IHC 22C3 pharmDx Interpretation Manual - NSCLC. Agilent DAKO

9. Munari E, Zamboni G, Marconi M, et al. PD-L1 expression heterogeneity in non-small cell lung cancer: evaluation of small biopsies reliability. Oncotarget. 2017, 8(52):90123-31

10. Roach C, Zhang N, Corigliano E, et al. Development of a Companion Diagnostic PD-L1 Immunohistochemistry Assay for Pembrolizumab Therapy in
Non-Small-cell Lung Cancer. Appl Immunohistochem Mol Morphol. 2016, 24(6):392-7

11. Marchetti A, Barberis M, Franco R, et al. Multicenter Comparison of 22C3 PharmDx (Agilent) and SP263 (Ventana) Assays to Test PD-L1 Expression for NSCLC Patients to Be Treated with Immune Checkpoint Inhibitors. J Thorac Oncol. 2017, 12(11):1654-63

12. Travis WD, Brambilla E, Burke AP, Marx A, Nicholson AG. WHO Classification of Tumours of the Lung, Pleura, Thymus and Heart. 2015. International Agency for Research on Cancer, Lyon, France

13. Mazieres J, Drilon A, Lusque A, et al. Immune checkpoint inhibitors for patients with advanced lung cancer and oncogenic driver alterations: results from the IMMUNOTARGET registry. Ann Oncol. 2019, 30(8):1321-8. 